\title{
Septa Design for a Prostate Specific PET Camera
}

\author{
Jinyi Qi, Jennifer S Huber, Ronald H Huesman, William W Moses, Stephen E Derenzo, Thomas F Budinger
}

\begin{abstract}
The recent development of new prostate tracers has motivated us to build a low cost PET camera optimized to image the prostate. Coincidence imaging of positron emitters is achieved using a pair of external curved detector banks. The bottom bank is fixed below the patient bed, and the top bank moves upward for patient access and downward for maximum sensitivity. In this paper, we study the design of septa for the prostate camera using Monte Carlo simulations. The system performance is measured by the detectability of a prostate lesion. We have studied 17 septa configurations. The results show that the design of septa has a large impact on the lesion detection at a given activity concentration. Significant differences are also observed between the lesion detectability and the conventional noise equivalent count (NEC) performance, indicating that the NEC is not appropriate for the detection task.
\end{abstract}

\section{INTRODUCTION}

Prostate cancer has a prevalence and diagnostic rate similar to breast cancer, with 360,000 new cases diagnosed each year and two million men affected by the disease in the United States. Prostate cancer suspicion is typically based on an elevated prostate-specific antigen (PSA) level or a suspicious node found during a digital rectal exam. Serum PSA values do not always correlate well with clinical diagnosis or outcomes. Palpation is subjective, insensitive and inexact. A new imaging technology for sensitive detection of early stage prostate cancer is needed to confirm initial diagnosis and help guide treatment decisions. In addition, a new method is needed to assess response shortly after treatment intervention.

The recent development of new prostate tracers has motivated us to build a low cost PET camera optimized to image the prostate [1]. Coincidence imaging of positron emitters is achieved using a pair of external curved detector banks, one placed above and one below the patient. Fig. 1 shows the transaxial view of the camera. The bottom bank is fixed below the patient bed, and the top bank moves upward for patient access and downward for maximum sensitivity. Each bank consists of two axial rows of 20 CTI ECAT HR+ block detectors ( $8 \times 8$ arrays of $4.5 \times 4.5 \times 30 \mathrm{~mm}^{3}$ BGO crystals), forming two arcs with a minor axis of $45 \mathrm{~cm}$ and major axis of $70 \mathrm{~cm}$. Our prostate camera has about one-fourth the number of detectors as in a conventional PET system because: (a) the patient is not fully encircled in 2D, (b) an elliptical shape is used instead of a circular one, and (c) the axial coverage is only $8 \mathrm{~cm}$. However, since the average distance to the detectors is approximately onehalf that of a conventional 2D PET system, the solid angle for a central source is approximately double for average-sized men.

This work is supported in part by Department of Defense grant number DAMD17-02-1-0081, by the Director, Office of Science, Office of Biological and Environmental Research, Medical Science Division of the U.S. Department of Energy under Contract No. DE-AC03-76SF00098, and by National Institutes of Health grant number R01 EB00194.

The authors are with the Department of Nuclear Medicine and Functional Imaging, Lawrence Berkeley National Laboratory, Berkeley, CA 94720, USA (telephone: 510-486-4695, e-mail: jqi@lbl.gov)
Thus, we expect to achieve improved detection efficiency at a lower cost.

Individual detector modules are angled to point towards the camera center near the prostate location in order to reduce penetration effects (in the detector) for annihilation photons originating in the prostate. This increases resolution selectively in the region of the prostate and is an unique feature for a non-circular camera geometry. Annihilation photons from other parts of the field of view (FOV) will suffer increased penetration effects, but these FOV regions are less important. Both detector banks can be tilted to image the prostate while minimizing attenuation (i.e., above the buttocks and below the stomach), but the gantry allows zero tilt for thin patients. A patient of average size is not fully encircled in 2D, which results in irregular and incomplete sampling due to the side gaps. Despite this incomplete sampling, we are able to reconstruct nearly artifact free images in the region of interest by using an iterative reconstruction algorithm [1].

The camera design includes inter-plane septa to reduce background events from random coincidences and from Compton scatter photons in the patient. Here we study the effect of different septa designs on the system performance using Monte Carlo simulations. Septa designs have been studied for conventional PET scanners [2], [3], [4], [5], [6], [7], as well as the coincidence gamma cameras [8], [9], [10]. In this work we focus on the specific application and special geometry of the prostate camera. The noise equivalent count (NEC), which is mostly used in previous studies, and a direct measure of the detectability of a prostate lesion in the data acquired by the camera are used in this study. Using the task-specific figure of merit, we can better assess the image quality. We also demonstrate the limitation of NEC for the prostate camera design.

\section{FIgURES OF MERIT}

A very popular figure of merit used to evaluate PET scanner design is the noise equivalent count, which is defined as

$$
\mathrm{NEC}=\frac{T^{2}}{T+S+k R}
$$

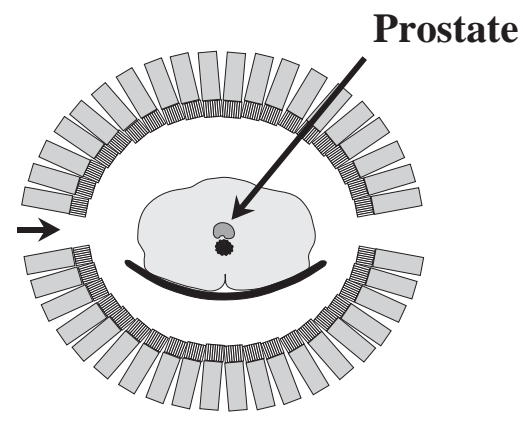

Fig. 1. A positron tomograph for prostate imaging. 
where $T, S$, and $R$ are total number of true coincidences, scattered events, and random events, respectively. Here we use $k=1$ assuming the randoms are not pre-subtracted and the expectation of the randoms can be estimated separately. NEC represents the overall signal-to-noise ratio (SNR) of the data set and is not specific to any local region. To study the image quality locally, some researchers proposed a local NEC [?], which is similar to (1) but only considers the lines of response that intersect with a specific region of interest when counting events.

Since our camera is designed specifically for imaging prostate tumors, we believe that it is more appropriate to measure system performance for this particular detection task. We choose a "signal-known-exactly, background-known-exactly" (SKE$\mathrm{BKE}$ ) task because the location of the prostate is relatively fixed and the background uptake is fairly uniform [11]. We use a prewhitening (PW) numerical observer, which is the ideal observer under Gaussian noise, to evaluate the lesion detectability.

Let $\boldsymbol{h}_{1}$ and $\boldsymbol{h}_{0}$ denote the expectations of the measurements with and without a lesion, respectively. The PW observer computes the following test statistic $\eta$ for a given data set $\boldsymbol{y}$

$$
\eta(\boldsymbol{y})=\left[\boldsymbol{h}_{1}-\boldsymbol{h}_{0}\right]^{\prime} \boldsymbol{\Sigma}^{-1} \boldsymbol{y}
$$

where $\boldsymbol{\Sigma}$ is the ensemble covariance matrix of $\boldsymbol{y}$. A decision whether there is a lesion or not is then made by comparing this statistic to a pre-selected threshold. The performance of the PW observer can be measured by the SNR, which is defined as

$$
\mathrm{SNR}^{2}=\frac{\left[\eta\left(\boldsymbol{h}_{1}\right)-\eta\left(\boldsymbol{h}_{0}\right)\right]^{2}}{\operatorname{var}[\eta(\boldsymbol{y})]} .
$$

PET data can be modeled as independent Poisson random variables with the expectation $\overline{\boldsymbol{y}}$ related to the tracer distribution $\boldsymbol{x}$ through an affine transform

$$
\bar{y}=\boldsymbol{P} \boldsymbol{x}+\boldsymbol{s}+\boldsymbol{r},
$$

where $\boldsymbol{P}$ is the projection probability matrix with the $(i, j)$ th element denoting the probability of detecting an event from the $j$ th voxel by the $i$ th LOR, $s$ and $r$ denote the expectation of the scattered and random events, respectively. Using this model, we can show that

$$
\begin{aligned}
\mathrm{SNR}^{2} & =\boldsymbol{f}^{\prime} \boldsymbol{P}^{\prime} \operatorname{diag}\left[\frac{1}{\bar{y}_{i}}\right] \boldsymbol{P} \boldsymbol{f} \\
& =\sum_{i} \frac{[\boldsymbol{P} \boldsymbol{f}]_{i}^{2}}{[\boldsymbol{P} \boldsymbol{x}+\boldsymbol{s}+\boldsymbol{r}]_{i}}
\end{aligned}
$$

where $f$ is the tracer uptake of a prostate lesion. The form in (4) is somewhat similar to that of NEC in (1). The major difference is that the SNR focuses only on the LORs that intersect with the lesion and the signal in each LOR is individually weighted by its variance.

When $\eta(\boldsymbol{y})$ is normally distributed, the SNR is related to the area under the ROC curve by the error function [12]. Therefore, we use (4) to measure the lesion detectability.

\section{Monte Carlo Simulation}

We use Monte Carlo simulation to estimate the true coincidence rate, scatters and randoms under each septa configuration.
TABLE I

PARAMETERS OF THE SIMULATED SEPTA DESIGNS

\begin{tabular}{c|c|c|c} 
No. & $\begin{array}{c}\text { Spacing } \\
\text { (crystal) }\end{array}$ & $\begin{array}{c}\text { thickness } \\
(\mathrm{mm})\end{array}$ & $\alpha$ \\
\hline 1 & 0.5 & 0.4 & 0.08 \\
2 & & 0.4 & 0.16 \\
3 & & 0.6 & 0.08 \\
4 & & 0.8 & 0.08 \\
\hline 5 & 1 & 0.4 & 0.16 \\
6 & & 0.4 & 0.32 \\
7 & & 0.8 & 0.08 \\
8 & & 0.8 & 0.16 \\
9 & & 0.8 & 0.32 \\
10 & & 1.2 & 0.08 \\
11 & & 1.2 & 0.16 \\
12 & & 1.6 & 0.08 \\
13 & & 1.6 & 0.16 \\
14 & & 2.4 & 0.04 \\
\hline 15 & 2 & 0.8 & 0.16 \\
16 & & 1.2 & 0.16 \\
17 & & 1.6 & 0.16 \\
\hline
\end{tabular}

The Monte Carlo simulation is conducted using the SimSET software $^{1}$. The current version of SimSET is limited to circular ring PET scanners. To study the special geometry of the prostate camera, we model the septa as part of the simulated object as shown in Fig. 2. Both Compton and photo-electric interactions are modeled in the patient body and septa. The patient body is an elliptical region with major and minor axes being $40 \mathrm{~cm}$ and $32 \mathrm{~cm}$, respectively, which correspond to the average size of six randomly selected male persons in our department. It has uniform activity and the attenuation coefficient of water. The shape of the septa is modeled as the difference of two elliptical regions with major and minor axes of the inner one being $57 \mathrm{~cm}$ and $32 \mathrm{~cm}$, respectively, and those of the outer one $(1+\alpha) 57 \mathrm{~cm}$ and $(1+\alpha) 32 \mathrm{~cm}$, respectively, where $\alpha$ is a constant ranging from 0.04 to 0.64 . The top and bottom axial planes contain perfect absorber to prevent photons from escaping from the axial openings so that the acceptance of a photon is completely determined by the septa. Therefore, we can use a ring detector surrounding the whole object and the resulting sensitivity is the same as that of the prostate camera. The thickness of the septa varies from $0.4 \mathrm{~mm}$ to $2.4 \mathrm{~mm}$. The spacing between the septa varies from half the crystal axial size $(2.4 \mathrm{~mm})$ to two crystal axial size $(9.7 \mathrm{~mm})$. Table I summaries the septa designs that we simulated.

True coincidence events and scattered events are binned separately. For all the results presented in this paper we use an energy threshold of $355 \mathrm{keV}$, except where noted otherwise. Singles rate is estimated by running the simulation in SPECT mode with the same number of photons. Random events are estimated from the singles rate by

$$
r_{k}=\tau \cdot \operatorname{singles}(i) \cdot \operatorname{singles}(j),
$$

where $\operatorname{singles}(i)$ and $\operatorname{singles}(j)$ are the singles rates of the two

\footnotetext{
${ }^{1}$ Available at http://depts.washington.edur/simset/html/simset_main.html
} 
detectors forming the $k$ th $\mathrm{LOR}$, and $\tau=12 n s$ is the coincidence timing window. All events are then corrected for deadtime as described in Section IV.

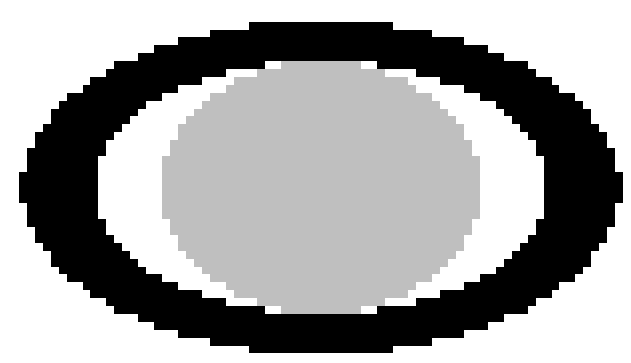

(a)

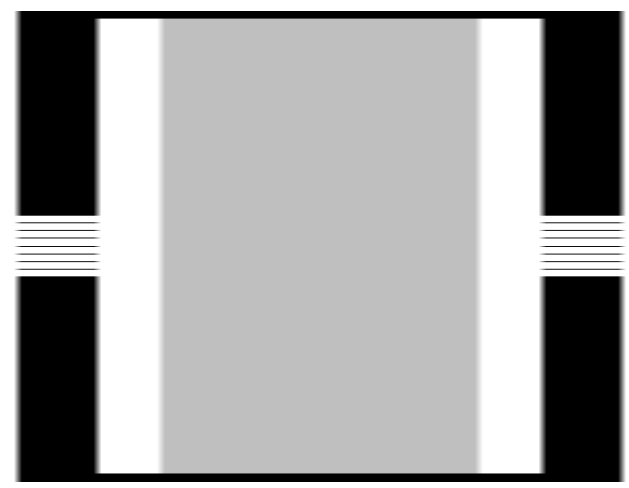

(b)

Fig. 2. Transaxial (a) and coronal (b) views through the center of the attenuation image used in the Monte Carlo simulation. The inner elliptical region is the patient body. The black band is the septa. The top and bottom axial planes contain perfect absorber to prevent photons from escaping from the axial openings. The parameters of septa shown in this figure are 2 crystals spacing, $1.2 \mathrm{~mm}$ thick, and $\alpha=0.16$.

\section{DEADTIME MODEL}

The deadtime of the camera is modeled similar to that described in [13]. Two parts of the deadtime are considered, one from the processing of single events at each detector block and the other from the coincidence processing circuit.

For each detector, the live time can be modeled as

$$
d_{i}=e^{-\tau_{\text {block }} N \cdot \overline{\operatorname{singles}}(i)}
$$

where $N$ is the number of detectors per detector block, $\overline{\text { singles }}(i)$ is the average singles rate of the individual detectors within the block, and $\tau_{\text {block }}$ is the time constant of the detector block. The sensitivity of the $k$ th LOR due to the detector block deadtime is then

$$
n_{k}=d_{i} * d_{j},
$$

where $i$ and $j$ are the indices of the two detectors forming the $k$ th LOR.

The system wide coincidence processing live time is

$$
\text { Coinc. Live }=e^{-\tau_{\text {sys }} \text { Coinc.Load }}
$$

where $\tau_{s y s}$ is the time constant, and Coinc.Load is the ideal count rate experienced by the coincidence processing circuit. It is calculated as

$$
\text { Coinc.Load }=\sum_{k}\left(t_{k}+s_{k}+2 r_{k}\right) n_{k},
$$

where $t_{k}, s_{k}$, and $r_{k}$ are the ideal count rates of the trues, scatters, and randoms in the $k$ th LOR, respectively. The factor of 2 accounts for the randoms in both prompt and delayed windows. The overall sensitivity of the $k$ th LOR due to the deadtime loss is $n_{k} *$ Coinc.Live.

\section{NORMALIZATION}

A $20 \mathrm{~cm}$ diameter, $19 \mathrm{~cm}$ long uniform cylinder with activity concentration of $0.06 \mu \mathrm{Ci} / \mathrm{cc}$ was scanned in an ECAT HR scanner (CTI PET systems, Knoxville, TN) in 3D mode. The measured singles rates are compared with that obtained from the Monte Carlo simulation of the same cylinder scan to calculate the detector efficiency. After scaling the simulation result with the calculated detector efficiency $(=0.49)$, the true coincidence, scatters, and randoms are compared. Fig. 3 shows the measured and simulated projection profiles. The simulated scatter profile matchs very well with the real measurement. The slight discrepancy between the true coincidence around the left peak is due to the attenuation of the patient bed, which is not modeled in the simulation. The comparison between the randoms calculated from singles rates and that measured using delayed window shows a nearly perfect match (a ratio of 1.0). The simulated event rate of trues plus scatters also matches the measured event rate well (a ratio of 0.97).

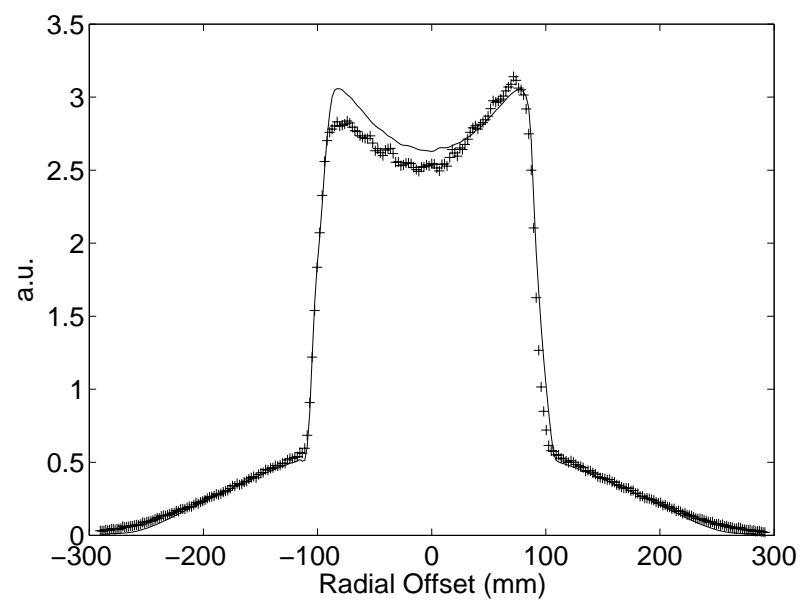

Fig. 3. Comparison between the measured ('+') and the simulated (solid line) projection profiles.

To estimate the time constants $\tau_{\text {block }}$ and $\tau_{\text {sys }}$ in the deadtime model, another uniform cylinder (the same geometry) with activity concentration of $0.6 \mu \mathrm{Ci} / \mathrm{cc}$ was scanned in the ECAT HR scanner. The system deadtime as reported by the scanner is about $23 \%$. The fitted time constants are $\tau_{\text {block }}=2.2 \mu s$ and $\tau_{\text {sys }}=118 n s$.

\section{RESULTS}

\section{A. Lesion detectability vs. NEC}

We studied 17 different septa configurations (Table I). Fig. 4(a) shows the lesion detectability at different activity lev- 
els for all the designs. The simulated lesion is a $2.5 \mathrm{~cm}$ diameter sphere located at the center of the FOV. The lesion to background activity ratio is $2: 1$. For comparison, NEC performance is shown in Fig. 4(b). Both lesion detectability and NEC show that longer septa are preferred for high activity concentration due to the reduction of scatters and randoms.

In Fig. 5, we plot the lesion detectability and NEC at $0.1 \mu \mathrm{Ci} / \mathrm{cc}$ activity concentration (which is the expected typical patient dose) for all the designs. Design no.17 outperforms others in terms of both lesion detection and NEC. Hence it is the best septa configuration among all that we have studied. However, significant differences are noticeable between the relative performance of lesion detection and NEC among other septa configurations, indicating that NEC is not a good measure for lesion detection task.

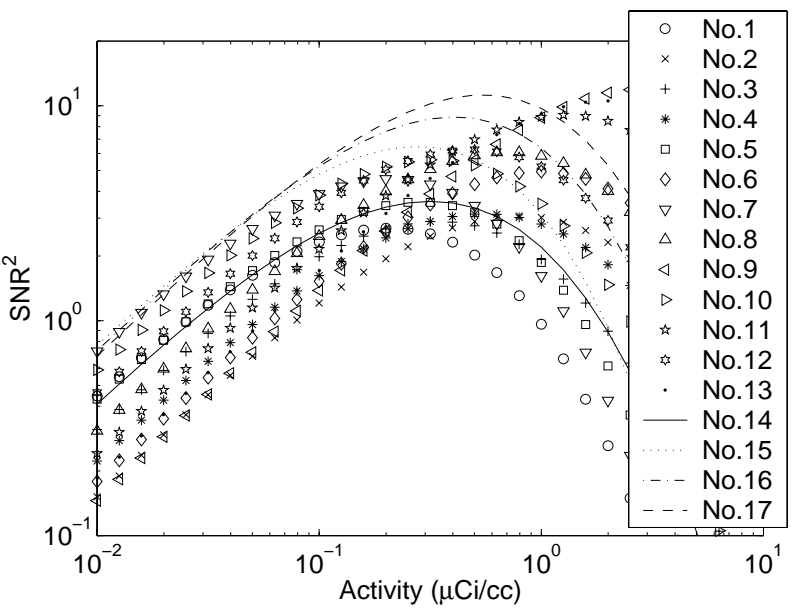

(a)

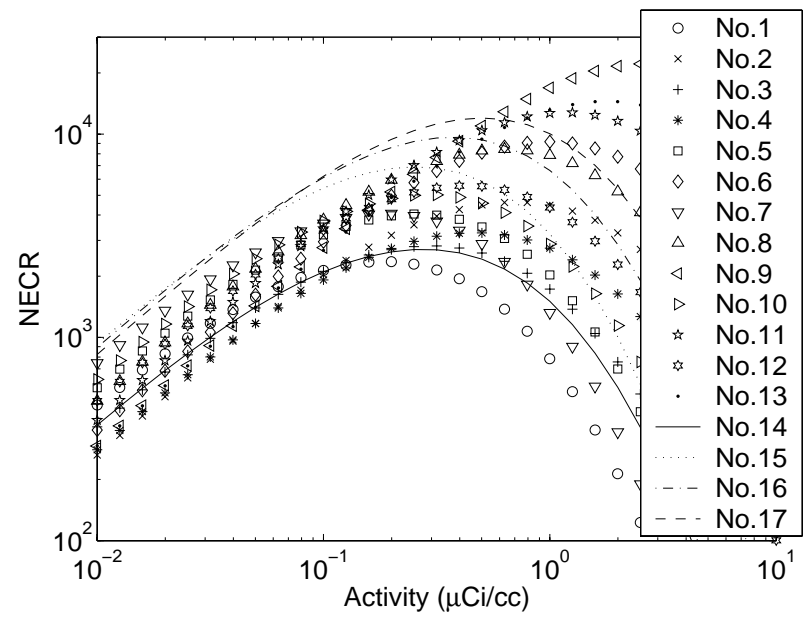

(b)

Fig. 4. Performance of 17 septa designs as a function of background activity levels. (a) lesion detectability plots; (b) NEC plots.

\section{B. Energy resolution}

We studied the effect of energy resolution on septa design by varying the energy threshold in the Monte Carlo simulation. Fig. 6(a) shows the performance of a system with perfect energy resolution where we set the energy threshold to $511 \mathrm{keV}$, and

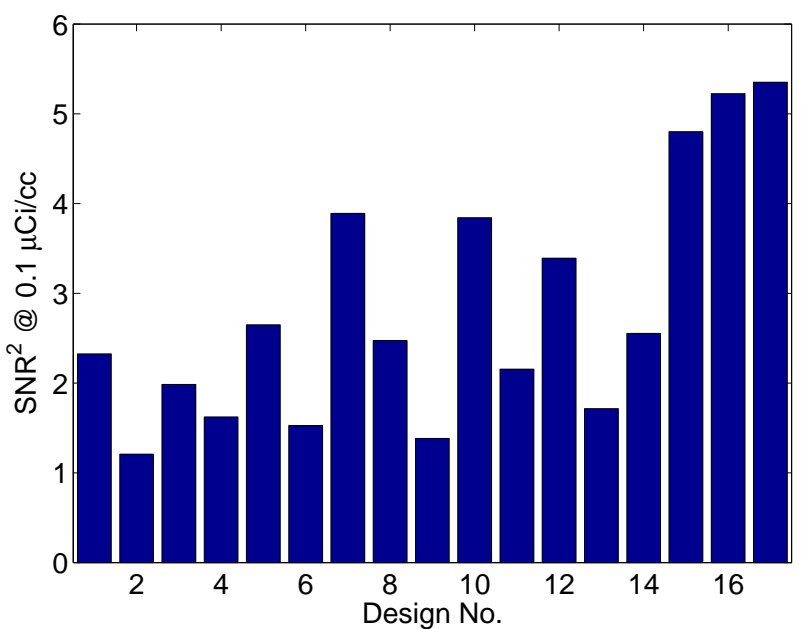

(a)

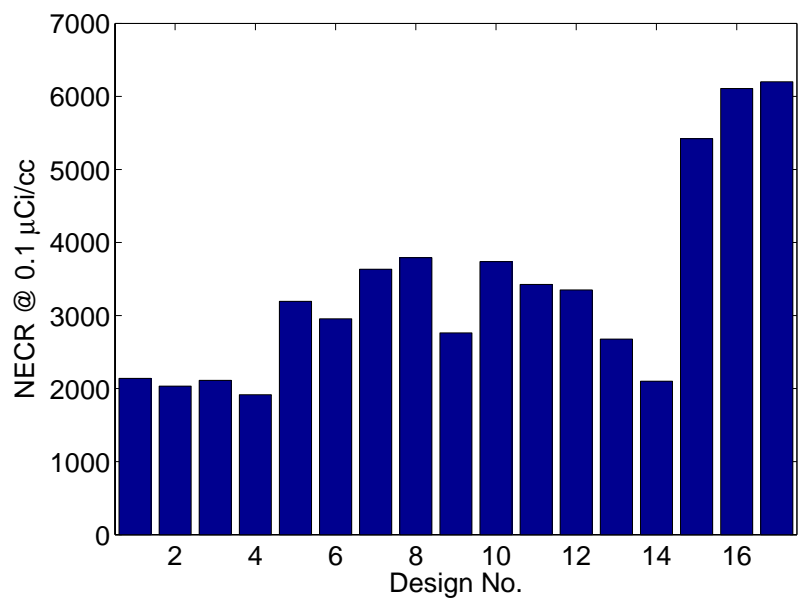

(b)

Fig. 5. Performance of all the septa designs at $0.1 \mu \mathrm{Ci} / \mathrm{cc}$. (a) lesion detectability; (b) NEC.

Fig. 6(b) shows the performance of a system with bad energy resolution where the energy threshold is $200 \mathrm{keV}$. Comparing the two plots, we found that at the same activity level, good energy resolution increases the lesion detectability and favors less septa due to the better rejection of events scattered inside patient body.

\section{Patient size}

To study the effect of patient body size on the septa design, we simulated a big patient using an ellipse with major and minor axes being $55 \mathrm{~cm}$ and $36 \mathrm{~cm}$, respectively. We compared three septa configurations as the trend will be similar for other designs. Fig. 7 compares the detectability of the prostate lesion inside a big patient vs. an average size patient. We found that for a large-size patient the lesion detectability reduces due to the increases in attenuation and background events, i.e., scatters and randoms, and hence longer and thicker septa is preferred.

\section{CONCLUSION}

We have conducted Monte Carlo simulations to study the effect of septa configuration on lesion detection. The results clearly show that a properly designed septa can improve lesion 


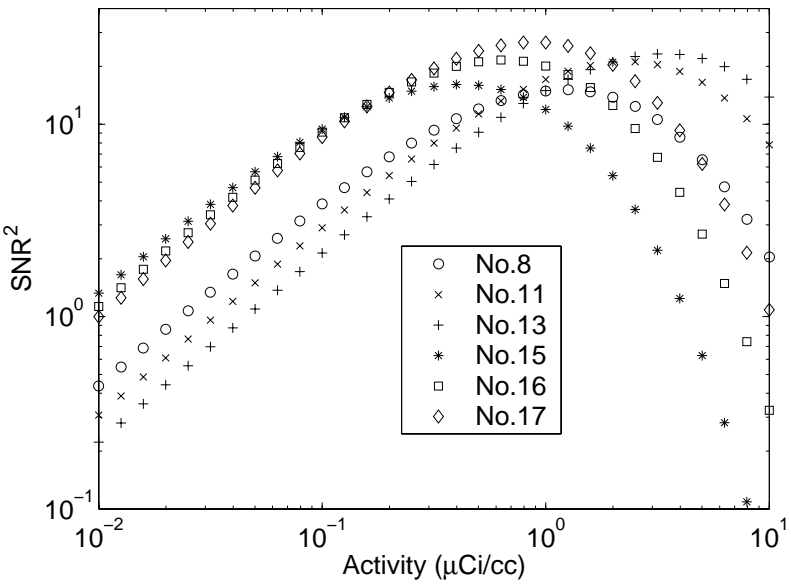

(a)

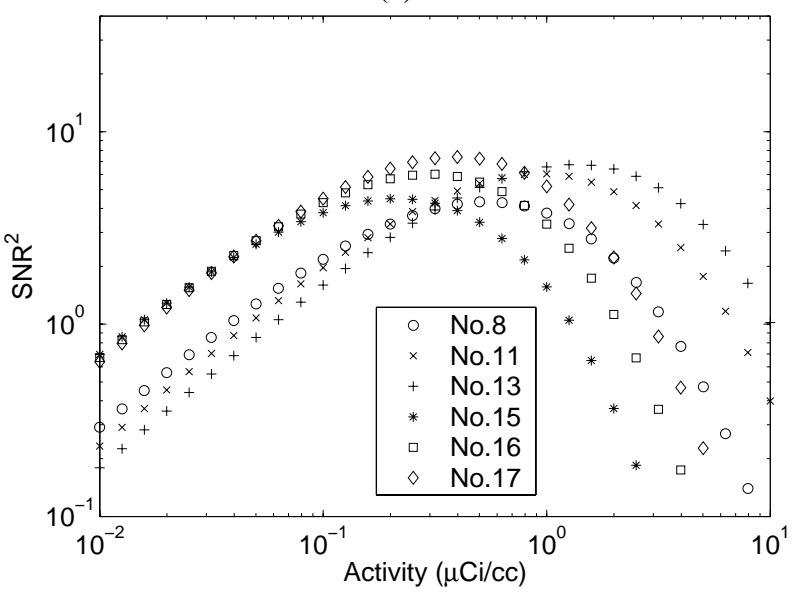

(b)

Fig. 6. Plots of lesion detectability with different energy threshold: (a) energy threshold $=511 \mathrm{keV}$; (b) energy threshold $=200 \mathrm{keV}$.

detection, although we cannot claim that we have found the optimal septa configuration due to the limited samples that we have studied. In future work we will continue the search for the optimal septa design for the prostate specific camera. We will construct several near optimal septa designs and compare their performance using real measurements.

\section{ACKNOWLEDGMENTS}

The authors would like to thank Robert Harrison at the University of Washington Medical Center, and Eric Frey and Ben Tsui at the Johns Hopkins University for their help in the SimSET software.

\section{REFERENCES}

[1] J. Huber, S. Derenzo, J. Qi, W. Moses, R. Huesman, and T. Budinger, "Conceptual design of a compact positron tomograph for prostate imaging," IEEE Trans. Nucl. Sci., vol. 48, pp. 1506-1511, 2001.

[2] C. Thompson, "The effect of collimation on scatter fraction in multi-slice PET," IEEE Trans. Nucl. Sci., vol. 35, pp. 598-602, 1988.

[3] L. E. Adam, J. S. Karp, and G. Brix, "Investigation of scattered radiotion in 3D whole-body positron emission using Monte Carlo simulations," Phy. Med. Bio., vol. 44, pp. 2879-2895, 1999.

[4] R. Lecomte, "Analytical study of performance in a 3D PET scanner," Phy. Med. Bio., vol. 37, pp. 623-634, 1992.

[5] M. Aykac, J. Uribe, H. Baghaei, H. Li, Y. Wang, Y. Liu, T. Xing, and W.-H.

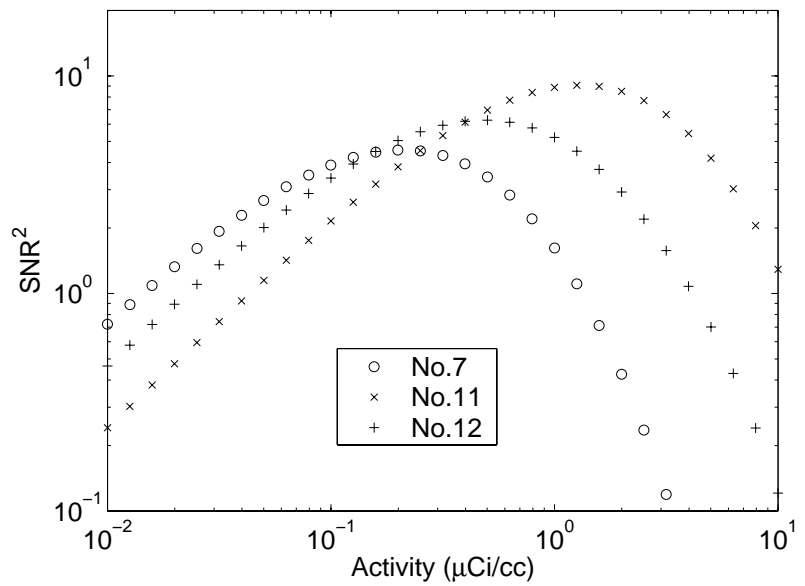

(a)

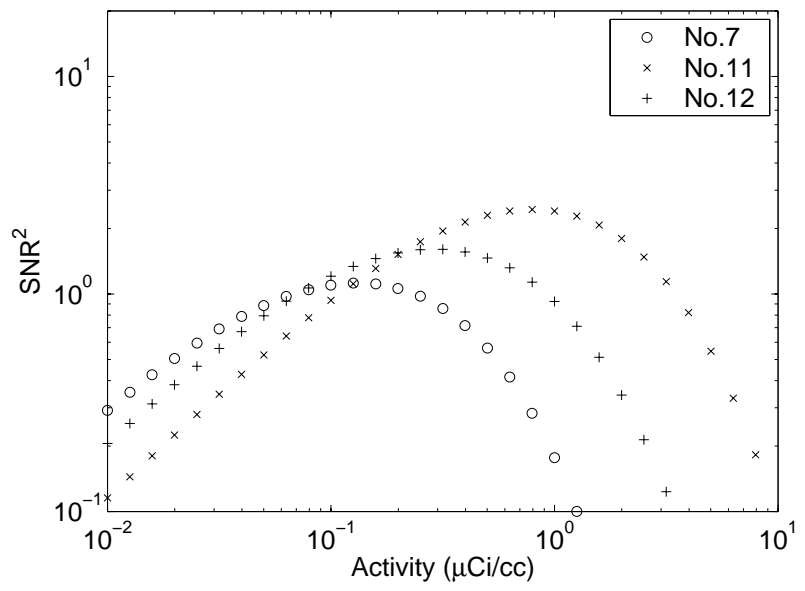

(b)

Fig. 7. Performance of three septa designs for detecting a prostate lesion in an average size patient (a) and a big patient (b).

Wong, "Septa design study for volumetric imaging in positron emission tomography," IEEE Trans. Nucl. Sci., vol. 49, pp. 2097-2102, 2002.

[6] T. Hasegawa, E. Tanaka, T. Yamashita, M. Watanabe, T. Yamaya, and H. Murayama, "A Monte Carlo simulation study on coarse septa for scatter correction in 3-D PET," IEEE Trans. Nucl. Sci., vol. 49, pp. 2133-8, 2002.

[7] H. Baghaei, W. H. Wong, J. Uribe, H. Li, M. Aykac, Y. Wang, Y. Liu, T. Xing, and R. Farrell, "Brain lesion detectability studies witha high resolution PET operating in no-septa and partial septa configurations," IEEE Trans. Nucl. Sci., vol. 50, pp. 1364-1369, 2003.

[8] C. J. Groiselle, Y. D’ Asseler, J. A. Kolthammer, C. G. Matthews, and S. J. Glick, "A Monte Carlo simulation study to evaluate septal spacing using triple-head hybrid PET imaging," IEEE Trans. Nucl. Sci., vol. 50, pp. 1339-46, 2003.

[9] T. C. Rust and D. J. Kadrmas, "Survey of parallel slat collimator designs for hybrid PET imaging," Phy. Med. Bio., vol. 48, pp. N97-104, 2003.

[10] S. J. Glick, C. J. Groiselle, J. Kolthammer, and R. Z. Stodilka, "Optimization of septal spacing in hybrid PET using estimation task performance," IEEE Trans. Nucl. Sci., vol. 49, pp. 2127-32, 2002.

[11] T. Hara, N. Kosaka, and H. Kishi, "PET imaging of prostate cancer using carbon-11-choline," J. Nucl. Med., vol. 39, pp. 990-995, 1998.

[12] H. H. Barrett, G. K. Abbey, and E. Clarkson, "Objective assessment of image quality. III. ROC metrics, ideal observers, and likelihood-generating functions," Journal of Optical Society of America A, vol. 15, pp. 15201525,1998

[13] L. Eriksson, K. Wienhard, and M. Dahlbom, "A simple data loss model for positron camera systems," IEEE Trans. Nucl. Sci., vol. 41, pp. 1566-1570, 1994. 